\title{
Correction to: Towards a Glaucoma Risk Index Based on Simulated Hemodynamics from Fundus Images
}

José Ignacio Orlando, João Barbosa Breda, Karel van Keer, Matthew B. Blaschko, Pablo J. Blanco, and Carlos A. Bulant

\section{Correction to:}

Chapter "Towards a Glaucoma Risk Index Based on Simulated Hemodynamics from Fundus Images"

in: A. F. Frangi et al. (Eds.): Medical Image Computing

and Computer Assisted Intervention - MICCAI 2018, LNCS 11071, https://doi.org/10.1007/978-3-030-00934-2_8

In the paper titled "Towards a glaucoma risk index based on simulated hemodynamics from fundus images", the acknowledgement has been updated.

The updated online version of this chapter can be found at https://doi.org/10.1007/978-3-030-00934-2_8 